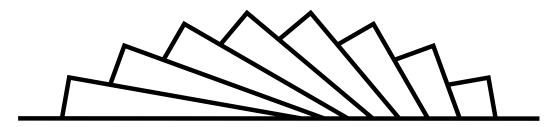

\title{
Soluciones Innovadoras Locales para la Reducción de la Pobreza
}

\author{
INNOVATIVE LOCAL SOLUTIONS FOR POVERTY REDUCTION
}

\section{RESUMEN}

La panorámica mundial muestra grandes deficiencias económicas y sociales, presentando una situación de marcada desigualdad social, pobreza, inequidad en la repartición de riqueza y de los ingresos, desempleo, subvaloración de la fuerza de trabajo, entre otros fenómenos sociales. La falta de oportunidades educativas, dentro de un sistema económico en decadencia como lo es el capitalismo requiere soluciones innovadoras y prácticas, capaces de afrontar la dura problemática de millones de seres en condiciones de indigencia. La implementación de la autogestión social parece ser una alternativa viable para la falta de empleo, y para lograr una redistribución más equitativa de los recursos y de los ing resos buscando ante todo generar un mejoramiento en la calidad de vida, de quienes, en estos momentos dentro de una sociedad capitalista, son los más marginados, subvalorados y excluidos. Se trata de recuperar ante todo una forma de participación en los asuntos que les pertenece, y que les atañen, es decir participar en las soluciones de sus necesidades insatisfechas bajo el principio de la subsidiariedad consagrado inicialmente en el preámbulo del tratado constitutivo de la Unión Europea según el cual las decisiones que afectan a los ciudadanos se deben adoptar de la forma más cercana posible a los mismos y su delegación a niveles o poderes más elevados solo deben hacerse por razones probadas de interés común.

Palabras clave: pobreza, innovación, localidad, subsidiariedad, autogestión.

\section{Julio Armando Rodríguez Ortega ${ }^{1}$}

${ }^{1}$ Doctor en Derecho de la Universidad Nacional de Colombia. Docente- investigador de la UPTC Contacto, julioarmand007@gmail. com. https://orcid.org/0000-0001-7427-1653

Fecha de recepción: 15/06/2019 Fecha de aprobación: 27/05/2020 
Citar este artículo así:

Rodríguez Ortega , J. A. (2021). Soluciones innovadoras locales para la reducción de la pobreza. Justicia, sociedad y derecho, $36-46$.

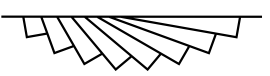

\begin{abstract}
The global panorama shows great economic and social deficiencies, presenting a situation of marked social inequality, poverty, inequality in the distribution of wealth and income, unemployment, undervaluation of the labor force, among other social phenomena. The lack of educational opportunities, within an economic system in decline such as capitalism requires innovative and practical solutions, capable of facing the harsh problems of millions of people in conditions of indigence. The implementation of social self-management seems to be a viable alternative for the lack of employment, and to achieve a more equitable redistribution of resources and income, seeking above all to generate an improvement in the quality of life of those who, at this time of a capitalist society, they are the most marginalized, undervalued and excluded. The aim is to recover first of all a form of participation in the issues that belong to them, and that concern them, that is, to participate in the solutions of their unmet needs under the principle of subsidiarity initially enshrined in the preamble of the Treaty establishing the European Union according to which decisions affecting citizens should be adopted as close as possible to them and their delegation to higher levels or powers should only be made for reasons of common interest.
\end{abstract}

Keywords: poverty, innovation, locality, subsidarity, self-management. 


\section{INTRODUCCIÓN}

Las características propias del estado Colombiano, tienen que ver con la diversidad de su geomorfología, la presencia de tres cordilleras, la cercanía y límites con dos mares, una red infinita de ríos como el Magdalena y el amazonas con sus afluentes, montañas, llanos, selvas, bosques y una diversidad climática incomparable que solo se puede equipar a la diversidad de razas y culturas que entre comunidades indígenas, afros y peninsulares que determinan su diversidad, que en el campo geográfico incluye diferentes territorios, regiones y comunidades.

La complejidad del mapa etnográfico y de los diferentes grupos humanos frente al desarrollo y sus cosmogonías hace necesaria la interpretación desde la administración pública de las realidades locales y regionales, por fuera de cualquier pretensión de homogeneidad que limite la posibilidad de la autodeterminación de sus comunidades; y el papel de la administración pública que se ha limitado a la reproducción de un proyecto de desarrollo que nunca ha tenido en cuenta las potencialidades de las comunidades locales y la misma complejidad de esa realidad local, haciendo del abandono Estatal no solo un problema de monopolio de la fuerza sino un atentado en contra del reconocimiento de un arraigo que no se puede ignorar y que requiere la interpretación de las realidades locales y los esquemas de desarrollo desde lo local, como acertadamente lo describe, Fernando Rodríguez en su disertación, sobre la encrucijada de la descentralización en Colombia. (Rodriguez Casallas, 2016).
Cuál es la viabilidad y cuáles los componentes para formular un plan estratégico orientado a la creación de empresas auto gestionadas que den viabilidad a soluciones innovadoras locales para la reducción de la pobreza y para la exigibilidad de los derechos sociales en bien de la comunidad con la participación del Estado, de las empresas, en el contexto de su responsabilidad social y los trabajadores, en su respectiva comunidad, en aras de lograr objetivos globales de desarrollo social y Bienestar general buscando ante todo una forma de participación en los asuntos que les pertenece, y que les atañen; es decir, participar en las soluciones de sus necesidades insatisfechas bajo el principio de la subsidiariedad.

A esta situación abiertamente se le puede considerar de crisis e incertidumbre, la cual está llevando a millones de seres humanos a la desesperanza, y al progresivo aumento de la pobreza. El crecimiento registrado en los últimos años, como consecuencia de la globalización solo ha favorecido a unos pocos y las desigualdades económicas tienen su reflejo en todos los componentes del sistema social, en la educación, en los valores y en la calidad de vida de todos los seres humanos.

\section{SOLUCIONES INNOVADORAS}

La panorámica mundial muestra grandes deficiencias económicas y sociales, presentando una situación de marcada desigualdad social, pobreza, inequidad en la repartición de riqueza y de los ingresos, desempleo, subvaloración de la fuerza de trabajo, entre otros fenómenos sociales. La falta de oportunidades educativas, 
dentro de un sistema económico en decadencia como lo es el capitalismo requiere soluciones innovadoras y prácticas, capaces de afrontar la dura problemática de millones de seres en condiciones de indigencia. La implementación de la autogestión social parece ser una alternativa viable para la falta de empleo, y para lograr una redistribución más equitativa de los recursos y de los ingresos buscando ante todo generar un mejoramiento en la calidad de vida, de quienes, en estos momentos dentro de una sociedad capitalista, son los más marginados, subvalorados y excluidos.

Es evidente la necesidad de proponer nuevas ideas, nuevos conceptos y sobre todo nuevas teorías económicas, que permitan encontrar propuestas de solución frente a los viejos e insuperables problemas de la pobreza, incorporando factores institucionales, iniciativa privada, participación de las universidades y las empresas, todo esto en un esfuerzo conjunto, para consolidar la responsabilidad social de todos los entes comprometidos en las comunidades locales, asumiendo roles solidarios y eficaces frente a su comunidad.

\section{EMPRESAS AUTO GESTIONADAS}

La autogestión social que se está proponiendo en esta investigación puede servir para llenar estos vacíos, de forma innovadora y sin tener que recurrir a un cambio drástico en el sistema económico, si se utiliza uno los recursos más subvalorados del sistema económico que es la fuerza de trabajo, en la solución de las propias necesidades recurso que puede generar una nueva perspectiva económica y social para la generación de una nueva época de cambios y de aprovechamiento más eficiente de la población improductiva y cesante que solo tiene expectativas de un trabajo formal y dependiente. La autogestión social se convierte en un instrumento de lucha contra la inequitativa distribución de la riqueza y en una alternativa viable para acceder a un estilo de vida más digno y a la solución de las propias necesidades.

Es necesario por lo tanto identificar, caracterizar y proponer los componentes, recursos y procesos viables para formular programas innovadores locales orientados a la reducción de la pobreza, particularmente la creación de empresas auto gestionadas y cogestionadas con la participación del estado en el marco de la eficacia y cumplimiento de los derechos sociales por mandato constitucional, lo mismo que la empresa en el marco de su responsabilidad social y los trabajadores insertados en sus comunidades, como sujetos y protagonistas de su propio desarrollo.

En igual sentido se trata de formular el marco teórico de los procesos innovadores locales para la reducción de la pobreza en el contexto de los objetivos del milenio formulados en el llamado PACTO GLOBAL de las naciones Unidas. Así mismo Identificar el desarrollo actual del pacto global y los objetivos de desarrollo sostenible en países o ciudades que en la actualidad impulsan programas innovadores locales para la reducción de la pobreza a través de empresas auto gestionadas y cogestionadas en el marco de los derechos sociales; de la responsabilidad social empresarial y de los fines sociales del Estado. 
En tal sentido se trata de identificar caracterizar y proponer los componentes, recursos y procesos de los programas innovadores locales para la reducción de la pobreza, particularmente la creación de empresas auto gestionadas y cogestionadas con la participación del estado en el marco de la eficacia y cumplimiento de los derechos sociales por mandato constitucional, lo mismo que la empresa en el marco de su responsabilidad social y los trabajadores insertados en sus comunidades, como sujetos y protagonistas de su propio desarrollo.

Los programas innovadores locales para la reducción de la pobreza y la responsabilidad social empresarial formulada en los objetivos del milenio contenidos en el pacto global de las Naciones Unidas no pueden estar en cabeza solo de la empresa, sino que deben comprometer, al Estado, a la comunidad, a los trabajadores y sus organizaciones, mediante acciones solidarias, que involucren el concepto de capitalismo solidario en la creación de proyectos comunitarios orientados a la solución de problemas sociales relevantes, cuyo protagonismo se realice a partir de la autogestión social y el efectivo ejercicio de los derechos sociales.

La búsqueda de innovaciones a nivel local se basa en que la mayor parte de las personas en estado de pobreza son portadores de una potencial oferta de fuerza de trabajo, que no cuenta con oportunidades por encontrarse en una sociedad precaria de iniciativas y emprendimiento comunitario y porque no hay ningún interés de las empresas en su potencial productivo. Las actuales tendencias mundiales que involucran la responsabilidad social de la Empresa, no pueden implementarse desconociendo los fines esenciales del Estado y el objetivo fundamental de su actividad. En igual forma no se puede ignorar la eficacia de la participación de la comunidad cuando se aplican programas de cogestión y autogestión con fundamento en el principio filosófico de no entregar el pez sino enseñar a pescar y no incurrir en el criticado Estado de Bienestar o en un paternalismo que puede resultar dañoso e inconveniente a ultranza para la solución de la pobreza en el mundo.

El ciudadano en su hábitat local es el protagonista de la construcción de lo social, particularmente en los asuntos de interés general de su propia colectividad, cuando se busca lograr el bienestar de todos. Las sociedades serán ser más sólidas, cuando promuevan la organización de sus ciudadanos, para reducir sustancialmente los niveles de pobreza, expresar sus preferencias, sus capacidades y hacer valer sus derechos. Cuando esto sucede, las personas se hacen sujetos sociales, resulta más fácil solucionar sus problemas y sobre todo, buscar el reconocimiento y apoyo del Estado, frente a la crisis e incertidumbre económica mundial que se ha mencionado, todo lo cual forma parte de la interpretación de las realidades locales y los esquemas de desarrollo desde lo local.(Rodríguez Casallas, 2016).

Se pretende encontrar la manera como las comunidades locales se hagan parte de un proceso tendiente a lograr unos fines sociales y económicos, auto gestionados con un compromiso social, patrocinados por la mano del Estado, arraigando fundamentalmente el valor de lo 
público y su prevalencia sobre el simple interés de lo privado o de la ganancia. Se trata de lograr la participación de la sociedad como una fuerza organizada y con grandes metas en la solución de sus propias necesidades y problemas. Estas metas se pueden lograr buscando promover por parte del Estado la participación ciudadana, integrándolos para encontrar el equilibrio donde las partes puedan aportar sus potencialidades al crecimiento de las mismas, es decir fundando las políticas públicas sobre la participación ciudadana con un rol preponderante autogestionario.

Las empresas auto gestionadas y la exigibilidad de los derechos sociales constituyen un camino viable y atractivo para las reducción de la pobreza a nivel local y teniendo en cuenta que la administración autónoma y su organización es decir la gestión y la autogestión empresarial utilizan métodos, habilidades y estrategias a través de las cuales los partícipes de una actividad puedan dirigirse hacia el logro de sus objetivos con autonomía de gestión. Los programas innovadores locales para la reducción de la pobreza requieren el establecimiento de metas, planificación, programación, seguimiento de tareas, autoevaluación, auto intervención y autodesarrollo de tal forma que la autogestión como proceso ejecutivo en el contexto de un proceso de ejecución autónomo logre el apoderamiento de los individuos para que cumplan objetivos por sí mismos, tendientes a lograr el pleno desarrollo de la comunidad la superación de la pobreza y el mejoramiento de su calidad de vida.

La sociedad aquejada por el alto índice de pobreza pide con gran insistencia una redistribución de la riqueza y de los ingresos lo mismo que cambios sustanciales en la forma como se distribuyen los recursos en la sociedad, teniendo en cuenta que el mundo asiste hoy a la consolidación y expansión de una justicia social, afirmando los derechos humanos y considerando importantes tendencias actuales que asocian la justicia en la explicación del fenómeno de la pobreza y el hambre global, contrario a quienes piensan exclusivamente en términos de factores causales domésticos propios de las sociedades en las cuales ocurren, reto que asumen las naciones Unidas en el Pacto Global bajo la bandera de la responsabilidad social, formulando los objetivos del milenio.

El concepto de justicia social, ya no se sustenta en las consideraciones constitucionales, ni en el concepto de soberanía estatal, sino en la persona humana y en la armonización de las legislaciones nacionales con los organismos de la justicia internacional, que particularmente en materia de derechos humanos constituye en el presente siglo el mayor reto que enfrentan los sistemas jurídicos del mundo. Se asocia la justicia en la explicación del fenómeno de la pobreza y el hambre global, contrario a quienes piensan como se afirmó antes exclusivamente en términos de factores causales domésticos propios de las sociedades en las cuales ocurren.

Se hace necesario promover procesos de autogestión local y de autoayuda, basados en las propias premisas de la población afectada. Existe una tendencia a tratar de resolver necesidades locales con soluciones fuera de lugar, creando una mayor dependencia en vez de autonomía y autosuficiencia. Enfrentados a grandes 
desafíos que impone la sociedad moderna, en la lucha por alcanzar una mejor calidad de vida para los seres humanos que respete y permita la permanencia de los sistemas naturales, es relevante que cada grupo humano, a distintos niveles se pueda desarrollar a su modo con creatividad y siendo recursivos. Se requieren alternativas de solución a sus necesidades cotidianas, otras formas diferentes de vida acordes con su realidad social, cultural y ambiental que permitan alcanzar altos niveles de autoabastecimiento, fortalecer el sentimiento comunitario y empoderar a estos grupos para transformarse en actores directos y diseñadores de su propio desarrollo desde una perspectiva local, integral y sustentable. (Casado Cañeque, 2006).

\section{EL CAPITALISMO SOLIDARIO}

La creación de empresas auto gestionadas no son otra cosa que la construcción social de numerosas asambleas y comunidades, concebidas como una respuesta espontánea a la crisis, económica y social propia de nuestro tiempo. Estos emprendimientos auto gestionados y cogestionados sostienen hoy la utopía de un desarrollo económico alternativo al del mercado capitalista que bien podría denominarse el camino hacia un capitalismo solidario, por medio de programas innovadores locales, que permitan la superación de la pobreza en el marco de la exigibilidad de los derechos sociales.

Se trata en esencia de una nueva economía social pues la autogestión y la cogestión no son un fin ni un medio sino un modo de actuar investigar y expresar la coherencia entre estos y aquellos, en términos organizativos, y con referencia tanto a la crítica teórico-práctica de lo existente, como a la propuesta de estructuras sociales alternativas. El hecho es que la autogestión, es una metodología organizativa de signo social si se aceptan plenamente todos los presupuestos y las implicaciones, en profundidad y en extensión con el concurso del Estado en el cumplimiento de los Derechos sociales.

Cuando se estudian las condiciones necesarias para que cada individuo pueda ser, verdaderamente, sujeto y no objeto de las alternativas que le conciernen, es cuando, por necesaria coherencia, se amplía el campo de aplicación de la autogestión y de la Co-gestión del angosto microcosmos de la fábrica a todas las esferas y todos los niveles de la vida social. Se pretende encontrar modelos alternativos de sociedad global con poder socializado, con una dimensión cultural, en la que se introducen criterios de juicio valores y criterios cognoscitivos modos de seleccionar y organizar los componentes de la comunidad para transformarlos en acción. La autogestión y la búsqueda de soluciones locales abre una alternativa en el cuerpo social, entre gestores y gestionados, pues la autogestión puede reconocerse y realizarse solo en la democracia directa, en el contexto de los derechos sociales, reconocidos a nivel universal.

Cuál es la viabilidad y cuales los componentes para formular un plan estratégico orientado a la creación de empresas auto gestionadas que den viabilidad a soluciones innovadoras locales para la reducción de la pobreza y para la exigibilidad de los derechos sociales en 
bien de la comunidad con la participación del Estado, de las empresas, en el contexto de su responsabilidad social y los trabajadores, en su respectiva comunidad, en aras de lograr objetivos globales de desarrollo social y Bienestar general buscando ante todo una forma de participación en los asuntos que les pertenece, y que les atañen, es decir participar en las soluciones de sus necesidades insatisfechas bajo el principio de la subsidiariedad.

\section{EL PRINCIPIO DE LA SUBSIDIARIEDAD}

Este principio señala que las decisiones que afectan a los ciudadanos se deben adoptar de la forma más cercana posible a los mismos y su delegación a niveles o poderes más elevados solo deben hacerse por razones probadas de interés común. Esta filosofía para actuar hace irreemplazable el proceso de autogestión y cogestión social para coordinar todos los recursos disponibles orientados a conseguir determinados objetivos particularmente la satisfacción de las necesidades básicas insatisfechas gracias al impulso que brindan para el desarrollo los diversos tipos de emprendimientos auto gestionados, a nivel local y comunitario.

Se pretende identificar, caracterizar y proponer los componentes, recursos y procesos de los programas innovadores locales para la reducción de la pobreza, particularmente la creación de empresas auto gestionadas y cogestionadas con la participación del estado en el marco de la eficacia y cumplimiento de los derechos sociales por mandato constitucional, lo mismo que la empresa en el marco de su responsabilidad social y los trabajadores insertados en sus comunidades, como sujetos y protagonistas de su propio desarrollo.

La investigación se ha propuesto en primer lugar formular el marco teórico de los procesos innovadores locales para la reducción de la pobreza en el contexto de los objetivos del milenio formulados en el llamado PACTO GLOBAL de las naciones Unidas, para lo cual se hace necesario identificar el desarrollo actual del pacto global y los países o ciudades que en la actualidad impulsan programas innovadores locales para la reducción de la pobreza a través de empresas auto gestionadas y cogestionadas en el marco de los derechos sociales; de la responsabilidad social empresarial y de los fines sociales del Estado.

Los programas innovadores locales para la reducción de la pobreza y la responsabilidad social empresarial formulada en los objetivos del milenio contenidos en el pacto global de las naciones Unidas no pueden estar en cabeza solo de la empresa, sino que deben comprometer, al Estado, a la comunidad, a los trabajadores y sus organizaciones, mediante acciones solidarias, que involucren el concepto de capitalismo solidario en la creación de proyectos comunitarios orientados a la solución de problemas sociales relevantes, cuyo protagonismo se realice a partir de la autogestión social y el efectivo ejercicio de los derechos sociales.

La problemática social y económica cada vez más acentuada en el contexto de la globalización y del neoliberalismo económico, y se evidencia el aumento de la pobreza, la miseria, el desempleo, la falta de oportunidades educativas y laborales, lo mismo que 
las graves carencias en materia de salud y bienestar de las comunidades, que viven hoy una dramática confrontación entre la modernidad y el atraso, que por situaciones de inequidad, no pueden abrirse camino hacia el desarrollo, porque se encuentran seriamente limitadas sus potencialidades en un mundo competitivo propio del capitalismo salvaje.

La adopción de acciones solidarias auto gestionadas no paternalistas, pueden contribuir a la solución global de esta problemática, si se crean proyectos comunitarios en que el capital se coloque al servicio de proyectos sociales auto gestionados y cogestionados que utilicen los recursos presentes en la comunidad, en las empresas y en el mismo estado, dejando atrás el viejo modelo estatista proteccionista del Estado de Bienestar que se debe convertir en el esfuerzo mancomunado de sus propios protagonistas, en la construcción de tejido social, a partir del cual haya oportunidades para todos, redistribución reconocimiento y ejercicio de los derechos sociales fundamentales. La relación capital-trabajo imperante en las Sociedades Actuales no desaparece en la empresa autogestionaria, sino que se mantiene en la medida en que el socio-trabajador es también empresario y se sumerge en la práctica empresarial orientada a la transformación social y política de la sociedad, mediante proyectos sociales destinados a satisfacer necesidades específicas y solventar las carencias a que se enfrentan las comunidades locales.

El concepto de autogestión en la actualidad se refiere a la búsqueda de formas de organización radicalmente nuevas de la vida económica, social y política normalmente se les llama empresas de trabajo asociado, en las cuales los trabajadores actúan como gestores y organizadores de la misma a nivel administrativo y capital con el fin de lograr una mayor integración de bienes y servicios. El papel del estado no solo se resume a la financiación económica, también técnica y de modos de proveer materias primas y la vigilancia de que las mismas sean utilizadas de manera productiva. como objetivos específicos el estado busca además de los ya mencionados, generar trabajo de manera natural, fortalecer el mercado interno, colaborar para que siempre haya un respaldo financiero e institucional, despeñar un rol de tutor de dichas empresas para monitorear su funcionamiento.

En Latinoamérica se están abriendo espacios para que las universidades tengan participación activa y colaboren con las empresas auto gestionadas que si bien no hacen aportes económicos si ayudan en la prestación de servicios tecnológicos y científicos más específicamente la transferencia de información y en la elaboración de un diagnóstico para saber cuáles son las necesidades insatisfechas y el potencial material y humano que se puede poner en ejercicio para solucionar tales necesidades si como la capacidad de sus trabajadores y las investigaciones que se pueden hacer para llevar a un óptimo mejoramiento de la calidad de vida de las mismas.

\section{OBJETIVOS DE DESARROLLO DEL MILENIO}

En vista de los niveles alarmantes en indicadores tales como la pobreza extrema, la inequidad, el acceso al agua potable -entre otros- en un número importante de 
naciones, para el año 2000 se aprueba la Declaración del Milenio por medio del voto afirmativo de 189 estados parte de las Naciones Unidas. En esta Declaración se enuncia los Objetivos del Milenio (ODM), que expresan la voluntad colectiva de lograr la paz y condiciones de vida dignas para todos los seres humanos. A partir de ese momento, los objetivos del milenio -compuestos por 8 objetivos y 21 indicadores- se convirtieron en un referente para medir los avances en materia de desarrollo humano (El espectador.com, 2014).

Ante esta situación Colombia no es la excepción, de modo que la consecución de los ODM influenció de manera directa e indirecta en el diseño de políticas públicas, programas y estrategias gubernamentales que buscaban mejorar indicadores como una mejor distribución de la riqueza, el acceso universal a la educación primaria, el alivio de la pobreza extrema, la lucha contra el hambre y la mortalidad infantil, entre otros. Siendo los ODM un marco de referencia sobre mejoramiento de condiciones y calidad de vida, la implementación de programas y políticas que de manera directa o indirecta aborden esos temas contribuyen a la consecución de aquellos.

Sin embargo, y a pesar de que los ODM están pensados desde un punto de vista gubernamental, se sabe que difícilmente un estado puede disponer de todos los recursos, infraestructura y recurso humano para llevar a cabo tan titánica tarea. Si bien pueden mencionarse casos de países con los elementos ya mencionados en las cantidades necesarias para cumplir esta tarea por su cuenta, la realidad indica que los países que no poseen los elementos suficientes para implementar estos programas a cuenta propia son precisamente los países que poseen las brechas más amplias entre el deber ser y el ser de estos indicadores (Casado, 2006). De este modo, puede comprenderse que el gobierno de estos países requiera de apoyo adicional para cumplir con los compromisos adquiridos al sumarse a la Declaración del Milenio.

Tradicionalmente se ha considerado que el apoyo para este tipo de programas debe provenir de entes externos, siendo la cooperación internacional la principal fuente de recursos y esfuerzos para complementar los esfuerzos gubernamentales. Sin embargo, hoy en día es necesario reconocer la importancia de otros actores, sobre todo a nivel interno (nacional, regional y local), que pueden aportar de manera más efectiva no solo al cumplimiento de estos objetivos, sino en general al diseño y ejecución de cualquier proyecto - política que deba ser implementada y afecte de una u otra manera a la sociedad. Dentro de esos actores, se ha aludido constantemente a la importancia de involucrar a estamentos de la sociedad tales como las organizaciones comunitarias, la comunidad educativa, los líderes religiosos, entre otros. Sin embargo, para los proyectos relacionados directamente con la calidad de vida, existe un sector cuya inclusión podría generar más beneficios de lograrse de otra manera: se trata del empresariado.

De este modo, Austin y Chuu sugieren que el sector empresarial puede tomar un rol prominente en la implementación de estrategias para cumplir con los 
ODM y mencionan que la erradicación de la pobreza conlleva tres requisitos que únicamente cumple el sector empresarial. Estos requisitos son: a) la posibilidad de intervenir en niveles de escala, b) que el apoyo ofrecido permanezca o se sostenga en el tiempo y c) el aprovechamiento máximo de los recursos con respecto a las metas, es decir la eficiencia y la eficacia. Los desarrollos teóricos no fueron los únicos que incluyeron al sector empresarial como un actor de peso en la lucha contra la pobreza.

\section{EL PACTO GLOBAL}

En el seno de la ONU, nuevamente, se gestiona el Pacto Global Mundial, una iniciativa que genera directrices sobre una posible inclusión efectiva y eficaz del empresariado con respecto a la generación de bienestar en la sociedad en la que producen y ofrecen sus productos y servicios. El Pacto Global comprende 4 grandes temáticas: derechos humanos, estándares laborales, medio ambiente y lucha contra la corrupción. Asimismo, establece 10 principios orientadores de la gestión empresarial que corresponderían a un punto determinado de cada una de las temáticas ya mencionadas. Los objetivos se trazan en aras de asimilar los 10 principios en todas y cada una de las actividades de la empresa y llevar a cabo acciones que contribuyan a la consecución de los ODM (El espectador.com, 2014).

Si bien hasta este punto no es la primera ni última vez que se relaciona el Pacto Global con los ODM, sí es la única vez que se menciona de manera explícita (El espectador.com, 2014). Es decir, se invita a las empresas a sumarse al Pacto Global con el objetivo de aportar de manera directa sobre el bienestar de la sociedad en general y la consecución de los ODM. (Casado Cañeque, 2006) Teniendo en cuenta lo anterior, es interesante abordar los efectos del Pacto Global en Colombia: Según una nota publicada en El espectador.com (2013), el pacto opera desde el 2006, pero trabaja formalmente desde el 2009. Para agosto del 2013 más de 400 empresas colombianas o que operan en Colombia se habían sumado a la iniciativa que contempla temas tan diversos como la erradicación del trabajo infantil, el empoderamiento de la mujer y la educación responsable.

La metodología de trabajo consiste en mesas de discusión que se reúnen cada 45 días y que están compuestas tanto por empresas como por expertos. Asimismo, se generan guías con respecto a los temas tratados. Todo esto enmarcado en el presupuesto de la importancia del valor compartido -esto es, trabajo conjunto con los grupos de interés para añadir valor tanto a la gestión como al producto final- y la importancia de las alianzas para un trabajo efectivo.

Ahora, de qué manera el Pacto Global puede potencializar el aporte del empresariado con respecto al cumplimiento de los ODM? Si bien son temas que en realidad son bastante afines, es posible abordar puntos concretos de la inclusión del sector empresarial en la lucha contra la pobreza. De esta manera, es posible superar el marco de referencia de la Responsabilidad Social Empresarial tradicional, que, si bien genera beneficios a la comunidad, no resulta ni eficiente ni eficaz, teniendo en cuenta que usualmente se contemplan acciones concretas y que muchas ve- 
ces se limita a las donaciones, sin considerar que la empresa puede generar muchos más beneficios y a largo plazo.

En este sentido, es conveniente revisar la posición de la empresa con respecto a la sociedad y sus posibilidades de intervención. Andrés M. Gaviria y Ana Afonso Gallegos (2010), en su texto El impacto del sector privado a través de los negocios inclusivos en los objetivos de desarrollo del milenio en Colombia, reseñan los hallazgos de A. Vives con respecto a las incompatibilidades estructurales del sector empresarial con respecto a los ODM y de hecho, sugiere la creación de empleo por parte de estas empresas como una solución eficaz para superar estas incompatibilidades, sin ser esta la única opción. El autor reseñado sugiere el cuidado de las relaciones de los diferentes estamentos de la sociedad y la inclusión de las iniciativas privadas en estas para lograr mayor eficacia y correspondencia con las necesidades reales de la población beneficiaria.

Existen otras propuestas con respecto al papel del sector empresarial en la generación de bienestar a la sociedad de manera sostenida y sostenible. Para Casado Cañeque (2006), el reto consiste en la superación de la filantropía como motor de la Responsabilidad Social Empresarial y la integración de la política social en las actividades y lineamientos generales de la empresa. La comprensión del papel de los otros actores y su influencia en el desarrollo de los ODM permitiría tomar decisiones más acordes con lo que realmente necesita la comunidad afectada. Por ejemplo, las empresas de comercio al detal podrían obtener los productos agrícolas de los proveedores de la zona en la que está ubicado el establecimiento en vez de obtenerlos de otras zonas.

El aporte generado por estas empresas no debería entenderse únicamente en términos filantrópicos: el potencial de acción de una empresa es muy amplio y el impacto que esto puede generar sobre el bienestar de una comunidad es muy alto. La generación de empleo en zonas deprimidas, siempre y cuando ateniéndose al estilo de vida de la comunidad, puede dar cuenta del impacto positivo que puede generarse en esta.

Sin embargo, es la interacción de la empresa con los demás actores la que podría ofrecer mejores perspectivas con miras a una implementación de estrategias que resulte eficaz a la hora de promover el bienestar de la comunidad e indirectamente, la consecución de los ODM. El reconocimiento del potencial de los demás actores permitiría un trabajo conjunto fluido y adecuado con respecto a las condiciones y necesidades de la comunidad en la que existe y funciona el empresariado. La inclusión de la comunidad en la cadena de valor, por ejemplo, representa una alternativa que genera gran bienestar en la comunidad siempre y cuando las condiciones laborales sean favorables a los empleados.

La inclusión de la comunidad es una opción sumamente atractiva y en otros países y en Colombia pueden contarse múltiples casos favorables, en los que se generó beneficios tanto a los empresarios como a la comunidad (PNUD Colombia, 2008). Sin embargo, existen dificultades importantes para esta labor como la escasa presencia estatal, la presencia de grupos armados o la falta de infraestructura adecuada. 
Sin embargo, es importante recordar que la generación de alternativas adecuadas a la situación y necesidad específicas tiene su base en la comprensión del entorno y de los actores que en él se desempeñan. Asimismo, el trabajo directo con la comunidad es un paso importante pero no es el primero que debe asumir una empresa que desee aportar favorablemente a la sociedad: la asimilación de los 10 principios del Pacto Global y la inclusión de sus lineamientos en las políticas generales de la empresa facilitan ampliamente la transición hasta el momento en el que la empresa pueda generar bienestar y mejor calidad de vida en la comunidad de una manera sostenible y duradera.

\section{BIBLIOGRAFÍA}

Acción Social. (24 de 9 de 2009). Alianzas público-privada en el marco de la red juntos. Obtenido de: http://www.accionsocial.gov.co/ documentos/2980_1491_Manual_Operativo_JUNTOS_v05.pdf

Austin, J., \& Chu, M. (2006). Finding a new weapon to attack poverty. Obtenido de Revista: Harvard Review of Latin America.: http://www.drclas. harvard.edu/revista/articles/view_spanish/875

Avina. (26 de 2 de 2010). Negocios y mercados inclusivos: definición y marco conceptual para el trabajo de Avina. Obtenido de: http:// itemsweb.esade.es/wi/research/iis/pdfs_web/JornadaSEKN/DefenicionNI-MarcoconceptualAVINA.pdf

Casado Cañejo, F. (2006). El papel del sector privado en el logro de lo objetivos del milenio. Economía Exterior, 35, 27 - 33. 
Casado Cañejo, F. (2009). Lecciones aprendidas sobre la implicación del sector privado en el logro de los objetivos de desarrollo del milenio. En Prandi, M; \& Lozano, J; (Eds). ¿Pueden las empresas contribuir a los objetivos del milenio? Barcelona: UAB.

Cecodes. (10 de 9 de 2009). Los negocios inclusivos en Colombia. Obtenido de: http://www.cecodes.org.co/descargas/documentos_ni/libro_ ni_colombia_diciembre08.pdf>

Compartamos Colombia. (10 de 9 de 2009). Inventario de Aprendizaje y Experiencias: Principales Conclusiones de la Sistematización de Casos de NI en America Latina. Obtenido de: http/espanol.nextbillion.net/lib/assets/documents/Conclusiones

El Espectador. (2 de 8 de 2013). Obtenido de Pacto Global Colombia: Organizaciones comprometidas con los principios de la ONU.: http://www.elespectador.com/tomalapalabra/pacific-rubiales/ pacto-global-colombia

El espectador.com. (2 de 1 de 2014). Pacto Global Colombia: Organizaciones comprometidas con los principios de la ONU. Obtenido de: http://www.elespectador.com/tomalapalabra/pacific-rubiales/ pacto-global-colombia-organizaciones-156-articulo

Gutíerrez, R., \& Avella, L. (2006). Gutíerrez, R.; Avella L. F.; \& Villar, R. (2006). Aportes y Desafíos de la Responsabilidad Social empresarial en Colombia. Bogotá: Fundación Corona.

Karnani, A. (2005). The Mirage of Marketing to the Bottom of the Pyramid: How the Private Sector Can Help Alleviate Poverty. California Management Review, 49, 90-112.

Licandro, O. (2009). Negocios inclusivos: un aporte para su reflexión conceptual y metodológica. Segundo Foro de Responsabilidad Social Empresarial 
del MERCOSUR - Organizan InWEnt y UNESCO. Asunción: MERCOSUR - Organizan InWEnt y UNESCO.

Organización de las Naciones Unidas. (2004). Aplicación de la declaración del milenio. A/59/282. New York: ONU, 2004. .

Organización de las Naciones Unidas. (2 de 1 de 2010). Objetivos de desarrollo del milenio:. Obtenido de: http://www.un.org/spanish/ millenniumgoals/pdf/MDG_Report_2009_SP_r3.pdf

Programa de las Naciones Unidas para el Desarrollo en Colombia. (23 de 10 de 2009). Objetivos de Desarrollo del Milenio en Colombia y el Sector Privado. Obtenido de: http://www.pnud.org.co/img_ upload/333161646164616461646164/SECTOR\%20PRIVADO\%20VE

Rodríguez Casallas, D. F. (24 de 10 de 2016). La encrucijada de la descentralización en Colombia: "antecedentes, problemáticas y retos en la implementación de la descentralización en Colombia como alternativa de lucha en contra de la pobreza". Justicia, Sociedad y Derecho, 1, 88-113. doi:https://doi.org/10.24267/25009389.156

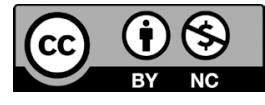

Esta obra está bajo una licencia de Creative Commons

Reconocimiento-NoComercial 4.0 Internacional 